\title{
Combined posterior contusion and penetrating injury in the pig eye. II. Histological features
}

\author{
ZDENEK GREGOR AND STEPHEN J. RYAN \\ From the Department of Ophthalmology, University of Southern California, \\ Estelle Doheny Eye Foundation, Los Angeles, California, USA
}

SUMmARY The main histological features observed after an experimental posterior contusion injury in the pig eye were haemorrhage from the choroidal vessels into the subretinal space and into the vitreous, uveal vessel dilatation, and an inflammatory reaction. These changes resolved during the first 2 weeks, and the subretinal haemorrhage was replaced by a fibrocellular membrane. The addition of a posterior penetrating injury resulted in fibrocellular proliferation at the wound site 6 days after injury and in formation of vitreoretinal traction membranes and subsequent traction retinal detachment. Posterior vitreous separation did not occur.

Penetrating eye injuries of the posterior segment which have a significant contusive component are frequently accompanied by an intraocular haemorrhage from the choroid which is more severe than if the eye were merely incised. ${ }^{12}$ The choroidal bleeding, which may occur at the time of injury ${ }^{3}$ or during subsequent vitrectomy, ${ }^{45}$ probably results from a combination of direct rupture of choroidal vessels and secondary choroidal vasodilatation. ${ }^{67}$

Knowledge of the natural history of an injury associated with choroidal engorgement would be of considerable importance in determining the optimum timing for surgical intervention. Ideally vitrectomy should be performed early enough to prevent the development of traction retinal detachment; on the other hand, with time, the danger of an uncontrollable intraocular haemorrhage lessens, making the procedure safer.

In the first part of this study we have described the production of choroidal bleeding by standard posterior contusion injury and the clinical features of its natural history with and without scleral penetration. ${ }^{2}$ In this paper we describe the sequence of histological changes following the experimental posterior contusion and penetrating injury in the pig eye.

\section{Material and methods}

As described in the preceding article, ${ }^{2} 38$ pig eyes were injured for the purposes of this study. In Group I, 10 eyes underwent a standard posterior contusion

Correspondence to Stephen J. Ryan, MD, Estelle Doheny Eye Foundation, 1355 San Pablo Street, Los Angeles, CA 90033, USA. injury alone; 10 eyes in group II had standard posterior penetrating injury alone; of these, 5 received intravitreal injection of autologous blood and 5 were injected with balanced salt solution (BSS). Group III consisted of 18 eyes that had a combined posterior contusion and penetrating injury; of these, 15 were injected with blood and 3 with BSS. The techniques used in the production of injuries is described in detail in the preceding paper. ${ }^{2}$

After the injury the animals were examined clinically and ultrasonographically at weekly intervals for up to 4 months. The eyes in group I were enucleated either immediately or at 4 months after the injury. The eyes in group II were enucleated at 4 months after the injury. The eyes in group III that had intravitreal blood injection were enucleated at 2-day intervals for the first 2 weeks, then at weekly intervals for the next month, and then at $8,10,14$, and 16 weeks after the injury. The 3 eyes in group III that had BSS injection were enucleated at 16 weeks after injury.

After enucleation a central corneal button was excised and the eyes were fixed in Karnovsky's fixative $(2.5 \%$ glutaraldehyde and $2 \%$ paraformaldehyde) in 0.1 molar cacodylate buffer $(\mathrm{pH}$ 7.2) for 24 hours. The eyes were then sectioned through the optic nerve and the inferior end of the wound for gross examination; each half was examined under the dissecting microscope, and drawings and photographs were made. The eyes were then dehydrated in alcohol, embedded in paraffin, sectioned, and stained in a routine manner for histopathological examination. 


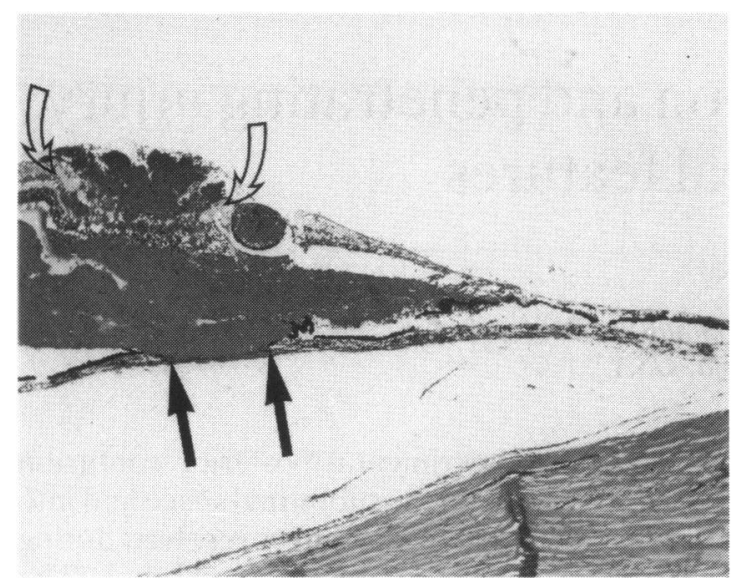

Fig.1A Anterior retina immediately after contusion. Bleeding occurred from the choroid and spread into the subretinal space and the vitreous (the choroid is artifactually detached). There is disruption of retinal pigment epithelium (black arrows) and focal retinal disruption (empty arrows). No dialysis occurred, and the marginal retinal vessel is intact.

\section{Results}

\section{GROUP I: STANDARD POSTERIOR CONTUSION} IN JURY

Gross examination of eyes enucleated immediately after contusion confirmed the presence of retinal whitening and wrinkling and subretinal and vitreous haemorrhage. The thickness of the ocular wall at the contusion site appeared unaltered.

Eyes enucleated 4 months after the injury showed a sharply demarcated area of hyperpigmentation and chorioretinal atrophy posterior to the contusion site. The vitreous was not separated from the retina and no retinal detachment was observed.

Ciliary body and choroid. Immediately after injury the ciliary processes adjacent to the contusion site appeared hyperaemic and the diameter of the anterior choroidal vessels was greater than that on the opposite side. In 4 eyes there were breaks in the choroidal vessel walls through which blood was seen tracking into the subretinal space and, through an area of retinal disruption, into the vitreous cavity.

Four months later there was proliferation of ciliary epithelium, which formed membranes consisting of multiple layers of elongated cells and spindle formations along the pars plana and the ciliary processes.

Vitreous. Other than vitreous haemorrhage the only abnormality observed immediately after injury was the avulsed vitreous base at the site of contusion. This was evidenced by a line of nonpigmented ciliary epithelial cells separated from the pigmented

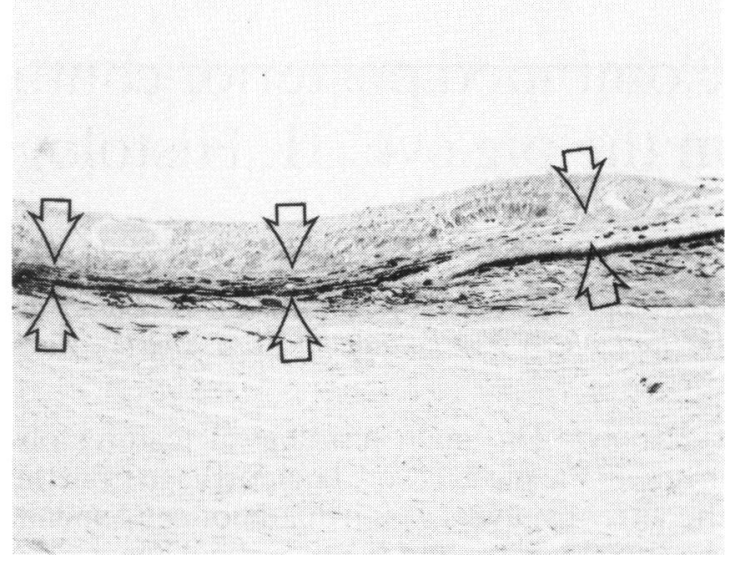

Fig. 1B Same area as in Fig. 1A 4 months after contusion. Retinal detachment is no longer present and a multilayered subretinal fibrocellular membrane replaces the subretinal blood (arrows).

layer and supported by the anterior vitreous base. At 4 months the vitreous appeared normal and was attached to the posterior retina.

Retina. Immediately after injury the anterior retina was shallowly detached, with numerous red blood cells and disrupted pigment epithelial cells in the subretinal space. All layers of the posterior retina were disrupted at the point of impact; subretinal haemorrhage was present and was continuous with choroidal and vitreous haemorrhage. Red blood cells were observed throughout the retinal layers. The marginal epiretinal vessel which courses along the ora serrata appeared to be intact (Fig. 1A). The posterior retina appeared normal.

Four months after injury the anterior retina at the impact site was thin and atrophic. The retinal pigment epithelium had undergone proliferation with the formation of multilayered fibrocellular membranes. Numerous pigmented macrophages were present throughout the anterior retina (Fig. 1B). There was no evidence of retinal detachment, and the posterior retina appeared normal.

\section{GROUP II: STANDARD POSTERIOR PENETRATING} INJURY

Examination of eyes which had blood injected 4 months previously revealed marked scar tissue at the inner aspect of the penetrating wound. This scar tissue distorted the ora serrata and caused traction, with anterior displacement of the peripheral retina at the wound site. In 3 eyes vitreous strands connected the wound site with the posterior pole, where 
the retina was thrown into multiple radial folds.

The vitreous was clear in all eyes but appeared to have separated from the posterior retina in 3 eyes.

The eyes which had BSS injected at the time of injury showed minimal fibrous proliferation at the wound site. The retina appeared normal, the vitreous was clear, and there were no signs of posterior vitreous separation.

Ciliary body and choroid. Microscopic examination of eyes which had blood injected at the time of injury confirmed the presence of exuberant fibrocellular proliferation at the wound site. Cellular proliferation appeared to follow planes of the incarcerated vitreous; the ora serrata was displaced anteriorly as though drawn towards the injury site.

In eyes which had BSS injected at the time of injury the fibrocellular proliferation was minimal and was confined to the pars plana.

Vitreous. The striking feature of eyes receiving blood injection was that, in spite of the apparent posterior vitreous separation noted in 3 eyes on clinical and gross histological examinations, on microscopic examination the cortical vitreous remained attached to the surface of the posterior retina in many areas. Such focal adhesions usually overlay large retinal vessels, though the vitreous was also attached to the tips of the posterior retinal folds. The remainder of the vitreous cortex appeared taut, spanning the sites of vitreoretinal adhesions in a linear fashion.

In eyes which had BSS injected the cortical vitreous was uniformly attached to the retinal surface.

Retina. The anterior retinal displacement in eyes with blood injection has been described above. The presence of retinal detachment, associated with the full-thickness retinal folds at the posterior pole, was confirmed by the presence of subretinal fluid, photoreceptor outer segment abnormalities, and macrophages in the subretinal space. The retinal pigment epithelium underlying these folds showed focal proliferation. Epiretinal cellular membranes were not present. Occasional pigmented macrophages were seen in the retina especially in the vicinity of major retinal vessels. Anteriorly the retinal architecture was undisturbed.

In the eyes injected with BSS the retinal architecture appeared normal; there were no inflammatory cells within the retinal layers.

GROUP III: THE COMBINED STANDARD POSTERIOR CONTUSION AND PENETRATING INJURY

During the first week the thickness of the eye wall in the region of the injury site was markedly increased. This was accounted for in part by scleral oedema and by a 2-fold increase in the thickness of the anterior choroid and the pars plana as compared with the area opposite the injury site. The thickness of the ocular walls became uniform, however, during the second week, with signs of fibrous proliferation appearing between the second and third weeks.

The appearance of a posterior vitreous detachment was observed in all blood injected eyes from the eleventh day after injury; this did not occur in the eyes which had BSS injected. Examination of the retina confirmed the presence of anterior and posterior retinal folds in all eyes in this group. No retinal folds or detachment occurred after a combined injury with injection of BSS.

Ciliary body and choroid. Vascular engorgement was noticeable during the first 4 days after injury. This engorgement was characterised by an increase in the diameter of the anterior choroidal vessels and by hyperaemia of the ciliary processes adjacent to the injury site. Similar changes, although much less marked, were also present on the side opposite the injury. By the end of the first week the size of the vascular channels on both sides appeared equal. As in the isolated contusion injury (group I), blood gained access to the subretinal space through breaks in the choroidal vessels and through areas of retinal disruption to the vitreous cavity.

Six days after injury fibroblasts were present on the inner aspect of the penetrating wound. These fibroblasts extended in a fan-like fashion along planes formed by condensations of incarcerated vitreous. They advanced anteriorly over the ciliary processes as well as posteriorly towards the ora serrata. By the end of the second week the fibroblastic proliferation from the wound had reached the peripheral retina and become incorporated in a fibrocellular membrane forming under the retina at the contusion site. Fibroblasts also proliferated along transvitreal bands which connected the perforating wound to posterior retinal folds.

One week after injury the nonpigmented ciliary epithelium appeared elongated, with cellular apices tilted towards the wound. A week later it began to proliferate, forming multiple layers of spindle-shaped cells over the pars plana. The ciliary processes adjacent to the wound appeared shortened and eventually became incorporated into the mass of proliferating fibroblasts.

Vitreous. In spite of the clinical impression of posterior vitreous separation, microscopy demonstrated a thin layer of cortical vitreous which remained adherent to the retina in numerous areas. The sites of vitreoretinal adhesion were usually on the crests of retinal folds and over retinal blood vessels; the posterior hyaloid spanned the distance between adjacent retinal folds (Fig. 2).

An acute inflammatory response occurred in the first 2 days after injury; this was characterised by a 


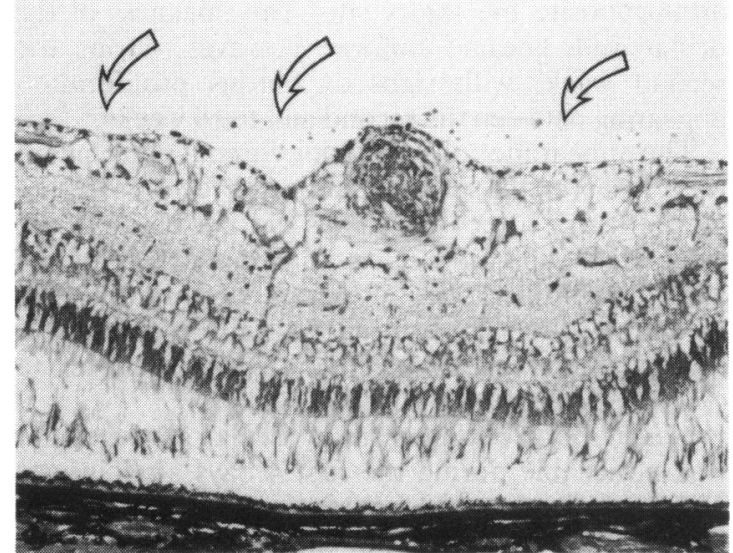

Fig. 2 Postequatorial retina 10 weeks after injury. Posterior vitreous face remains attached to the retinal surface and the vessels (arrows). Note the numerous inflammatory cells in the retinal capillary lumen and in the retina.

polymorphonuclear cell infiltration of the ciliary body and anterior vitreous. A week later macrophages predominated, and then gradually the overall number of inflammatory cells decreased.

Retina. Retinal detachment was confirmed on microscopic examination. Other notable histological features included the presence of haemorrhage and changes in the retinal architecture and the pigment epithelium.

Circumferential retinal folds adjacent to the injury site were present from the first day until the end of the second week after the injury. These folds appeared as shallow elevations of neurosensory retina, with the subretinal space filled with eosinophilic exudate and a large number of red blood cells and, later, macrophages. The subretinal fluid frequently extended as far as the equator (Fig. 3A).

Detachment of the posterior retina appeared as full-thickness retinal folds at the posterior pole from the eighth day after injury. In some eyes these folds increased progressively in size as they were drawn towards the penetrating wound by transvitreal membranes (Fig. 3B). In eyes followed up for 3 to 4 months a forward roll of peripheral retina on to the ciliary body was seen $180^{\circ}$ from the injury site.

Three to 6 weeks after injury a perivascular cuffing of monocytic cells was found in all layers of the retina.

The subretinal haemorrhage, which was present for the first 2 weeks after injury, appeared to have its origin from choroidal vessels. As in the contused eyes in group I, this was evidenced by continuity of the subretinal blood clot with large choroidal vessels through breaks in Bruch's membrane and the pigment epithelium. This haemorrhage also gained

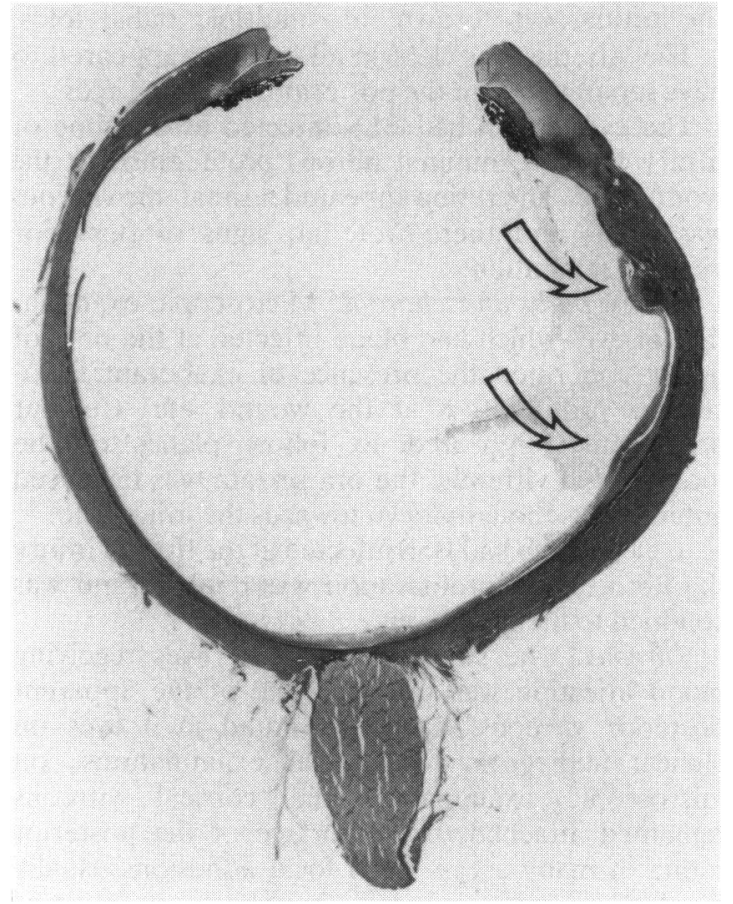

Fig. 3A Blood-filled circumferential retinal folds 6 days after injury; retinal folds extend just posterior to the equator. The posterior retina is attached.

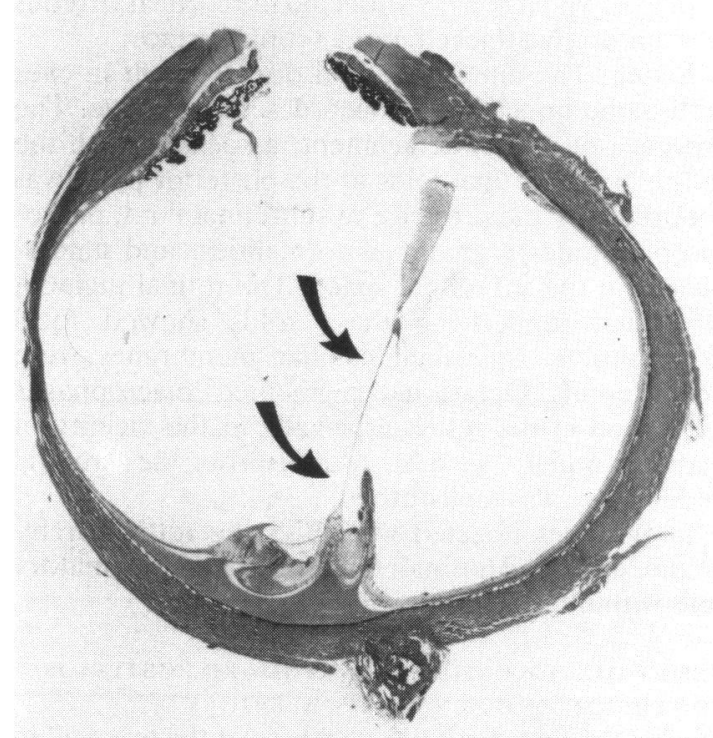

Fig. 3B Three weeks later than Fig. 3A there are high traction folds in the posterior pole. Transvitreal bands connect the penetrating wound to the retinal surface. The anterior retina is now attached. 
access to the vitreous through areas of retinal disruption at the contusion site posterior to the ora serrata.

Disruption of the retinal architecture at the contusion site was observed in the first 2 weeks after injury. It was accompanied by loss of outer segments, deep retinal haemorrhages, and folding of the outer nuclear layers in the equatorial retina adjacent to the injury site. Vacuolisation of the nerve fibre and ganglion cell layers, with disruption of the inner nuclear layer, were more prominent opposite the contusion site.

At the end of the first week after injury the pigment epithelium underlying areas of subretinal haemorrhage showed signs of proliferation, and at 2 weeks after injury the subretinal haemorrhage was completely replaced by a fibrocellular membrane. The subretinal membrane at the contusion site became continuous with the fibrous proliferation arising from the inner aspect of the penetrating wound (Fig. 1B). This subretinal membrane increased in size and became vascularised in the late stages of follow-up.

In the last 2 months of follow-up the retinal pigment epithelium on the side of the injury showed signs of proliferation characterised by formation of folds, loops, and multicellular sheaths. Occasionally the pigment epithelial cells were less pigmented and less granular, which was interpreted as macrophage transformation. These changes were observed often well posterior to the injury site, frequently extending as far as the posterior pole.

\section{Discussion}

In this study the main features of the contusion injury were the initial hyperaemia of the choroid and ciliary body at the impact site and the occurrence of intraocular haemorrhage, originating from the choroid. These changes were difficult to discern clinically, but histological study confirmed that the bleeding originated from choroidal vessels and gained access to the subretinal space and, usually, to the vitreous through gaps in Bruch's membrane and in the pigment epithelium and through disruption of the retina. Localised haemorrhagic retinal detachments, however, did not progress to rhegmatogenous or traction retinal detachment. Rather, the subretinal blood was in time replaced by a fibrocellular membrane which appeared to enhance the chorioretinal adhesion in this area. These findings correlate well with clinical ${ }^{8}$ and experimental ${ }^{9}$ studies, in which retinal detachment has not been observed to occur from retinal damage at the impact site. Nevertheless, it is recognised that delayed retinal detachment may occur due to retinal dialysis or retinal tears or as a result of vitreous traction locally or at sites distant from the injury. ${ }^{10}$

When we combined a penetrating injury with the contusive injury, " signs of vitreoretinal traction and traction retinal detachment became apparent. The main histological features of such an injury were the development of intravitreous fibrous proliferation from the penetrating wound and the formation of transvitreal traction bands. Within the first week of injury proliferating fibroblasts extended along condensations of the incarcerated vitreous anteriorly over the ciliary processes as well as posteriorly towards the ora serrata. Interestingly, the appearance of intravitreal cellular proliferation coincided with the absorption of the subretinal haemorrhage.

The anteroposterior vitreous traction produced full-thickness retinal folds at the posterior pole. The appearance was identical to that observed in the pig eyes which had suffered a penetrating injury with blood injection without the contusive component (group II), and it resembled the experimental traction retinal detachment produced previously in rabbit eyes. ${ }^{1213}$ Histological examination of the eyes in our study revealed that the cortical vitreous remained attached to the retinal surface throughout the followup period. The apparent posterior vitreous separation observed clinically and on examination of gross specimens seemed to have resulted from cleavage in the plane of the cortical vitreous, with the outermost layer of the peripheral vitreous remaining attached to the internal limiting membrane. Such attachments and subsequent vitreous membrane contraction may then have been responsible for vitreoretinal traction and, later, retinal detachment. In this respect the pig eye differs from the monkey and human eyes, both of which develop posterior vitreous separation after a penetrating injury with vitreous haemorrhage. The absence of posterior vitreous separation in the pig may also account for the relative lack of anterior and circumferential vitreous traction observed in human ${ }^{7}$ and primate ${ }^{10}$ eyes, in which an injury of a similar magnitude to that used in this study usually culminates in total retinal detachment.

Observations on this experimental animal model may be helpful in making clinical decisions on the timing of vitrectomy in injured human eyes. Immediately after injury engorgement of uveal vessels and inflammatory swelling of the otherwise relatively avascular pars plana make the entry of vitrectomy instruments into the eye hazardous and may result in an intraocular haemorrhage. In addition it has been observed clinically that changes in intraocular pressure may also lead to greater exudation and bleeding from the dilated and probably atonic ${ }^{6}$ choroidal vessels. It was noteworthy that in our experimental model these vascular 
changes began to abate by the end of the first week, which correlated well with clinical observations on human eyes. However, significant intraocular fibrocellular proliferation did not occur until the end of the first week after injury. This observation provides further support for delaying surgical intervention until the second week after a severe penetrating injury; during this time the risks of operative haemorrhage lessen, while the intraocular fibrosis has not yet become established.

To test this hypothesis we will perform a controlled trial of vitrectomy in the treatment of combined posterior contusion and penetrating injury in this model.

We thank Kate Borkowski and her colleagues for technical assistance and Sue Gertson for secretarial help.

This study was supported in part by NIH grants EY 02061 and EY 03040 (Dr Ryan).

\section{References}

1 Duke-Elder S, MacFaul PA. Concussions and contusions. In: Duke-Elder S, ed. System of ophthalmology. St Louis: Mosby, 1972: 14 (1): 73 .

2 Gregor Z, Ryan SJ. Combined posterior contusion and penetrating injury in the pig eye: $\mathrm{I}$. A natural history study. $\mathrm{Br} J$ Ophthalmol 1982; 66: 793-798.
3 Eagling EM. Perforating injuries involving the posterior segment. Trans Ophthalmol Soc UK 1975; 95: 335.

4 Faulborn J, Atkinson A, Olivier D. Primary vitrectomy as a preventive surgical procedure in the treatment of severely injured eyes. Br J Ophthalmol 1977; 61: 202-8.

5 Machemer R, Norton EWD. A new concept for vitreous surgery. 3. Indications and results. Am J Ophthalmol 1972; 74: 1034-56.

6 Duke-Elder S, MacFaul PA. Concussions and contusions. In: Duke-Elder S, ed. System of ophthalmology. St Louis: Mosby, 1972: 14 (1): 73.

7 Winthrop SR, Cleary PE, Minckler DS, Ryan SJ. Penetrating eye injuries: a histopathological review. Br J Ophthalmol 1980; 64: 8809-17.

8 Bloome MA, Ruiz RS, Russo CE, Salmonsen PC. Acute retinal necrosis. Ann Ophthalmol 1979; 11: 723-8.

9 Cox S. Retinal breaks caused by blunt nonperforating trauma at the point of impact. Trans Am Ophthalmol Soc 1980; 78: 414-66.

10 Weidenthal DT, Schepens CL. Peripheral fundus changes associated with ocular contusion. Am J Ophthalmol 1966; 62: 465-77.

11 Cleary PE, Ryan SJ. Method of production and natural history of experimental posterior penetrating eye injury in the rhesus monkey. Am J Ophthalmol 1979; 88: 212-20.

12 Cleary PE, Ryan SJ. Experimental posterior penetrating eye injury in the rabbit. I. Method of production and natural history. Br J Ophthalmol 1979; 63: 306-11.

13 Radtke ND, Tano Y, Chandler D, Machemer R. Simulation of massive periretinal proliferation by autotransplantation of retinal pigment epithelial cells in rabbits. Am J Ophthalmol 1981; 91: 76-87.

\section{Note}

\section{Colour Group}

The Colour Group (Great Britain) is holding a seminar on

'Acquired defects of colour vision' at the Institute of Ophthalmology, all day on 5 January 1983. Inquiries to Dr D. A. Palmer, Institute of Ophthalmology, Judd Street, London WC1H 9QS. 